\title{
HIERARCHICAL TRANSFORMER-BASED LARGE-CONTEXT END-TO-END ASR WITH LARGE-CONTEXT KNOWLEDGE DISTILLATION
}

\author{
Ryo Masumura, Naoki Makishima, Mana Ihori, Akihiko Takashima, Tomohiro Tanaka, Shota Orihashi \\ NTT Media Intelligence Laboratories, NTT Corporation, Japan
}

\begin{abstract}
We present a novel large-context end-to-end automatic speech recognition (E2E-ASR) model and its effective training method based on knowledge distillation. Common E2E-ASR models have mainly focused on utterance-level processing in which each utterance is independently transcribed. On the other hand, large-context E2E-ASR models, which take into account long-range sequential contexts beyond utterance boundaries, well handle a sequence of utterances such as discourses and conversations. However, the transformer architecture, which has recently achieved state-of-the-art ASR performance among utterance-level ASR systems, has not yet been introduced into the large-context ASR systems. We can expect that the transformer architecture can be leveraged for effectively capturing not only input speech contexts but also long-range sequential contexts beyond utterance boundaries. Therefore, this paper proposes a hierarchical transformer-based large-context E2E-ASR model that combines the transformer architecture with hierarchical encoder-decoder based large-context modeling. In addition, in order to enable the proposed model to use long-range sequential contexts, we also propose a large-context knowledge distillation that distills the knowledge from a pre-trained large-context language model in the training phase. We evaluate the effectiveness of the proposed model and proposed training method on Japanese discourse ASR tasks.
\end{abstract}

Index Terms - large-context endo-to-end automatic speech recognition, transformer, hierarchical encoder-decoder, knowledge distillation

\section{INTRODUCTION}

In the automatic speech recognition (ASR) field, end-to-end ASR (E2E-ASR) systems that directly model a transformation from an input speech to a text have attracted much attention. Main advantage of E2E-ASR systems is that they allow a single model to perform the transformation process in which multiple models, i.e., an acoustic model, language model, and pronunciation model, have to be used in classical ASR systems. In fact, E2E-ASR systems have the potential to perform overall optimization for not only utterance-level processing but also discourse-level or conversation-level processing.

Toward improving E2E-ASR performance, several modeling methods have been developed in the last few years. The initial studies mainly adopted connectionist temporal classification [1,2] and recurrent neural network (RNN) encoder-decoders [3, 4]. Recent studies have used the transformer encoder-decoder, which provided much stronger ASR performance [5, 6]. The key strength of the transformer is that relationships between the input speech and output text can be effectively captured using a multi-head self-attention mechanism and multi-head source-target attention mechanism.
These modeling methods have mainly focused on utterancelevel ASR in which each utterance is independently transcribed. Unfortunately, utterance-level ASR models cannot capture the relationships between utterances even when transcribing a series of utterances such as discourse speech and conversation speech. On the other hand, large-context E2E-ASR models, which take into account long-range sequential contexts beyond utterance boundaries, have received increasing attention. Previous studies reported that large-context models outperform utterance-level models in discourse or conversation ASR tasks [7, 8], and hierarchical RNN encoder-decoder modeling has been mainly introduced into the large-context E2E-ASR models. However, the transformer architecture has not yet been introduced into the large-context ASR systems. The transformer architecture is expected to be leveraged for effectively capturing not only input speech contexts but also long-range sequential contexts beyond utterance boundaries.

In this paper, we propose a hierarchical transformer-based largecontext E2E-ASR model that combines the transformer architecture with hierarchical encoder-decoder based large-context modeling. The key advantage of the proposed model is that a hierarchical transformer-based text encoder, which is composed of tokenlevel transformer encoder blocks and utterance-level transformer encoder blocks, is used to convert all preceding sequential contexts into continuous representations. In the decoder, both the continuous representations produced by the hierarchical transformer and input speech contexts are simultaneously taken into consideration using two multi-head source-target attention layers. Moreover, since it is difficult to effectively exploit the large-contexts beyond utterance boundaries, we also propose a large-context knowledge distillation method using a large-context language model [9.-13]. This method enables our large-context E2E-ASR model to use the largecontexts beyond utterance boundaries by mimicking the behavior of the pre-trained large-context language model. In experiments on discourse ASR tasks using a corpus of spontaneous Japanese, we demonstrate that the proposed model provides ASR performance improvements compared with conventional transformer-based E2EASR models and conventional large-context E2E-ASR models. We also show that our large-context E2E-ASR model can be effectively constructed using the proposed large-context knowledge distillation.

\section{RELATED WORK}

Large-context encoder-decoder models: Large-context encoderdecoder models that can capture long-range linguistic contexts beyond sentence boundaries or utterance boundaries have received significant attention in E2E-ASR [7, 8], machine translation [14 15], and some natural language generation tasks [16 17]. In recent studies, transformer-based large-context encoder-decoder models have been introduced in machine translation [18 19]. In addition, a fully transformer-based hierarchcal architecture similar to our transformer 
architecture was recently proposed in a document summarization task [20]. To the best of our knowledge, this paper is the first study that introduces the hierarchical transformer architecture into largecontext E2E-ASR modeling.

Knowledge distillation for E2E-ASR: For E2E-ASR modeling, various knowledge distillation methods have been proposed. The main objective is to construct compact E2E-ASR models by distilling the knowledge from computationally rich models [21-23]. Methods for distilling the knowledge from models other than ASR models into E2E-ASR models have also been examined recently. Representative methods are used to distill knowledge from an external language model to improve the capturing of linguistic contexts 24, 25]. Our proposed large-context knowledge distillation method is regarded as an extension of the latter methods to enable the capturing of all preceding linguistic contexts beyond utterance boundaries using large-context language models [9.13.

\section{HIERARCHICAL TRANSFORMER-BASED LARGE-CONTEXT E2E-ASR MODEL}

This section details our hierarchical transformer-based large-context E2E-ASR model that integrates the transformer encoder-decoder with hierarchical encoder-decoder modeling. Large-context endto-end ASR can effectively handle a series of utterances, i.e., conversation-level data or discourse-level data, while utterance-level end-to-end ASR handles each utterance independently.

In our hierarchical transformer-based large-context E2E-ASR model, the generation probability of a sequence of utterancelevel texts $\mathcal{W}=\left\{\boldsymbol{W}_{1}, \cdots, \boldsymbol{W}_{T}\right\}$ is estimated from a sequence of utterance-level speech $\mathcal{X}=\left\{\boldsymbol{X}_{1}, \cdots, \boldsymbol{X}_{T}\right\}$, where $\boldsymbol{W}_{t}=$ $\left\{w_{t, 1}, \cdots, w_{t, N_{t}}\right\}$ is the $t$-th utterance-level text composed of tokens and $\boldsymbol{X}_{t}=\left\{\boldsymbol{x}_{t, 1}, \cdots, \boldsymbol{x}_{t, M_{t}}\right\}$ is the $t$-th utterance-level speech composed of acoustic features. The notation $T$ is the number of utterances in a series of utterances, $N_{t}$ is the number of tokens in the $t$-th text, and $M_{t}$ is the number of acoustic features in the $t$-th utterance. The generation probability of $\mathcal{W}$ is defined as

$$
\begin{aligned}
P(\mathcal{W} \mid \mathcal{X}, \boldsymbol{\Theta}) & =\prod_{t=1}^{T} P\left(\boldsymbol{W}_{t} \mid \boldsymbol{W}_{1: t-1}, \boldsymbol{X}_{t}, \boldsymbol{\Theta}\right) \\
& =\prod_{t=1}^{T} \prod_{n=1}^{N_{t}} P\left(w_{t, n} \mid w_{t, 1: n-1}, \boldsymbol{W}_{1: t-1}, \boldsymbol{X}_{t}, \boldsymbol{\Theta}\right),
\end{aligned}
$$

where $\Theta$ is the model parameter set. Utterances from the 1 -st to $t-1$-th utterance is defined as $\boldsymbol{W}_{1: t-1}=\left\{\boldsymbol{W}_{1}, \cdots, \boldsymbol{W}_{t-1}\right\}$, and tokens from the 1-st to $n-1$-th token for the $t$-th utterance is defined as $w_{t, 1: n-1}=\left\{w_{t, 1}, \cdots, w_{t, n-1}\right\}$.

ASR decoding of a sequence of utterance-level texts from a sequence of utterance-level acoustic features using large-context endto-end ASR is achieved by recursively conducting utterance-level decoding. The ASR decoding problem for the $t$-th utterance is defined as

$$
\hat{\boldsymbol{W}}_{t}=\underset{\boldsymbol{W}_{t}}{\operatorname{argmax}} P\left(\boldsymbol{W}_{t} \mid \hat{\boldsymbol{W}}_{1: t-1}, \boldsymbol{X}_{t}, \boldsymbol{\Theta}\right),
$$

where $\hat{\boldsymbol{W}}_{1: t-1}$ are ASR outputs from the 1-st utterance to the $t-1$ th utterance. Therefore, $\hat{W}_{t}$ is recursively used for decoding the text of the $t+1$-th utterance.

\subsection{Network structure}

We construct our hierarchical transformer-based E2E-ASR model using a hierarchical text encoder, speech encoder, and text decoder.

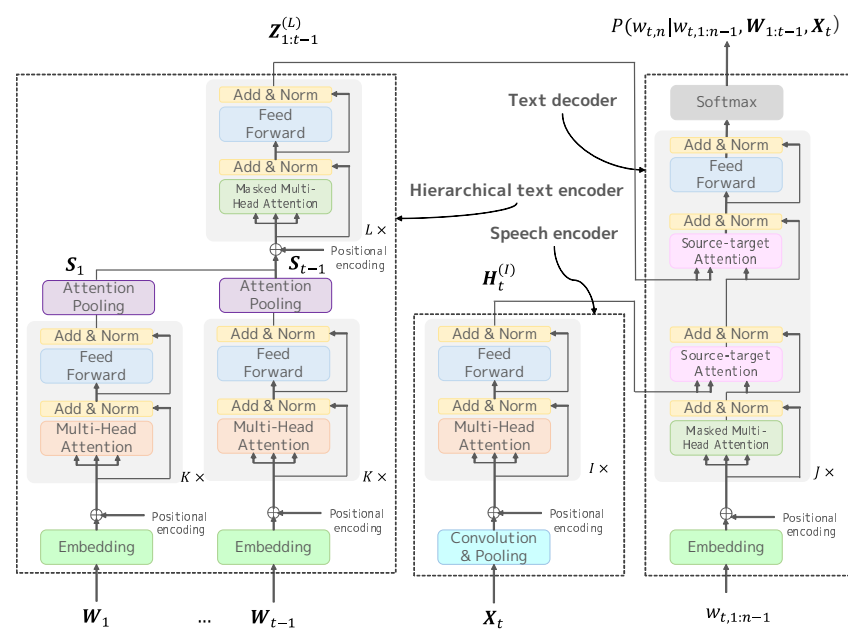

Fig. 1: Network structure of our hierarchical transformer based large-context E2E-ASR model.

Thus, we define the model parameter set as $\boldsymbol{\Theta}=\left\{\boldsymbol{\theta}_{\text {henc }}, \boldsymbol{\theta}_{\text {senc }}, \boldsymbol{\theta}_{\text {dec }}\right\}$ where $\boldsymbol{\theta}_{\text {henc }}, \boldsymbol{\theta}_{\text {senc }}$ and $\boldsymbol{\theta}_{\text {dec }}$ are the parameters of the hierarchical text encoder, the speech encoder and the text decoder, respectively. Figure 1 shows the network structure of the proposed model. Each component is detailed as follows.

Hierarchical text encoder: The hierarchical text encoder, which is constructed from token-level transformer blocks and utterancelevel transformer blocks, embeds all preceding pre-decoded text into continuous vectors. In fact, to perform ASR for the $t$-th utterance, we can efficiently produce the continuous vectors by only feeding the $t-1$-th pre-decoded text.

For the $t$-1-th pre-decoded text, the $n$-th token is first converted into a continuous vector $\boldsymbol{c}_{t-1, n}^{(0)} \in \mathbb{R}^{d \times 1}$ by

$$
\begin{gathered}
\boldsymbol{w}_{t-1, n}=\text { Embedding }\left(w_{t-1, n} ; \boldsymbol{\theta}_{\text {henc }}\right), \\
\boldsymbol{c}_{t-1, n}^{(0)}=\operatorname{AddPosEnc}\left(\boldsymbol{w}_{t-1, n}\right),
\end{gathered}
$$

where Embedding ( $)$ is a function to convert a token into a continuous vector and AddPosEnc () is a function that adds a continuous vector in which position information is embedded. The notation $d$ represents the hidden representation size. The continuous vectors $\boldsymbol{C}_{t-1}^{(0)}=\left\{\boldsymbol{c}_{t-1,1}^{(0)}, \cdots, \boldsymbol{c}_{t-1, N_{t}}^{(0)}\right\} \in \mathbb{R}^{d \times N_{t}}$ are embedded into an utterance-level continuous vector using $K$ transformer encoder blocks and an attention pooling layer. The $k$-th transformer encoder block creates the $k$-th hidden representations $C_{t-1}^{(k)} \in \mathbb{R}^{d \times N_{t}}$ from the lower layer inputs $\boldsymbol{C}_{t-1}^{(k-1)}$. The $t-1$-th utterance-level continuous vector $\boldsymbol{S}_{t-1} \in \mathbb{R}^{d \times 1}$ is produced by

$$
\begin{aligned}
& \boldsymbol{C}_{t-1}^{(k)}=\operatorname{TransformerEnc}\left(\boldsymbol{C}_{t-1}^{(k-1)} ; \boldsymbol{\theta}_{\text {henc }}\right), \\
& \boldsymbol{S}_{t-1}=\text { AttentionPooling }\left(\boldsymbol{C}_{t-1}^{(K)} ; \boldsymbol{\theta}_{\text {henc }}\right),
\end{aligned}
$$

where TransformerEnc() is a function of the transformer encoder block that consists of a multi-head self-attention layer and positionwise feed-forward network [5]. AttentionPooling () is a function that uses an attention mechanism to summarize several continuous vectors as one continuous vector [26].

Next, to take utterance-level sequential contexts into consideration, we embed utterance-level position information as

$$
\boldsymbol{Z}_{t-1}^{(0)}=\operatorname{AddPosEnc}\left(\boldsymbol{S}_{t-1}\right) \text {. }
$$


We then produce the $t$-1-th context-dependent utterance-level continuous vector from $\boldsymbol{Z}_{1: t-1}^{(0)}=\left\{\boldsymbol{Z}_{1}^{(0)}, \cdots, \boldsymbol{Z}_{t-1}^{(0)}\right\} \in \mathbb{R}^{d \times(t-1)}$ using $L$ utterance-level masked transformer encoder blocks. The $t-$ 1-th context-dependent utterance-level continuous vector $\boldsymbol{Z}_{t-1}^{(L)} \in$ $\mathbb{R}^{d \times 1}$ is computed from

$$
\boldsymbol{Z}_{t-1}^{(l)}=\text { MaskedTransformerEnc }\left(\boldsymbol{Z}_{1: t-1}^{(l-1)} ; \boldsymbol{\theta}_{\text {henc }}\right),
$$

where MaskedTransformerEnc () is a masked transformer encoder block that consists of a masked multi-head self-attention layer and position-wise feed-forward network. Finally, we construct vectors $\boldsymbol{Z}_{1: t-1}^{(L)} \in \mathbb{R}^{d \times(t-1)}$ by concatenating $\boldsymbol{Z}_{t-1}^{(L)}$ with pre-computed ones $\boldsymbol{Z}_{1: t-2}^{(L)} \in \mathbb{R}^{d \times(t-2)}$.

Speech encoder: The speech encoder converts input acoustic features into continuous representations, which are used in the text decoder. For transcribing the $t$-th utterance's input speech, the speech encoder first converts the acoustic features $\boldsymbol{X}_{t} \in \mathbb{R}^{f \times M_{t}}$ into subsampled representations $\boldsymbol{H}_{t}^{(0)}=\left\{\boldsymbol{h}_{t, 1}^{(0)}, \cdots, \boldsymbol{h}_{t, M_{t}^{\prime}}^{(0)}\right\} \in \mathbb{R}^{d \times M_{t}^{\prime}}$ as

$$
\begin{gathered}
\boldsymbol{H}_{t}=\text { ConvolutionPooling }\left(\boldsymbol{X}_{t} ; \boldsymbol{\theta}_{\text {senc }}\right), \\
\boldsymbol{h}_{t, m}^{(0)}=\operatorname{AddPosEnc}\left(\boldsymbol{h}_{t, m}\right),
\end{gathered}
$$

where ConvolutionPooling() is a function composed of convolution layers and pooling layers. The notation $M_{t}^{\prime}$ is the subsampled sequence length of the $t$-th input speech depending on the function. Next, the speech encoder converts the hidden representations $\boldsymbol{H}_{t}^{(0)}$ into $\boldsymbol{H}_{t}^{(I)} \in \mathbb{R}^{d \times M_{t}^{\prime}}$ using $I$ transformer encoder blocks. The $i$ th transformer encoder block creates the $i$-th hidden representations $\boldsymbol{H}_{t}^{(i)} \in \mathbb{R}^{d \times M_{t}^{\prime}}$ from the lower layer inputs $\boldsymbol{H}_{t}^{(i-1)}$ by

$$
\boldsymbol{H}_{t}^{(i)}=\operatorname{TransformerEnc}\left(\boldsymbol{H}_{t}^{(i-1)} ; \boldsymbol{\theta}_{\text {senc }}\right) \text {. }
$$

Text decoder: The text decoder computes the generation probability of a token from the hidden representations of the speech and all preceding tokens of not only the target utterance but also preceding utterances.

We detail the procedure of estimating the generation probability of the $n$-th token for the $t$-th utterance. We first convert preestimated tokens $w_{t, 1: t-1}$ for the $t$-th output text into continuous vectors $\boldsymbol{u}_{t, 1: n-1}^{(0)} \in \mathbb{R}^{d \times(n-1)}$ as

$$
\begin{gathered}
\boldsymbol{w}_{t, 1: n-1}=\operatorname{Embedding}\left(w_{t, 1: n-1} ; \boldsymbol{\theta}_{\mathrm{dec}}\right), \\
\boldsymbol{u}_{t, 1: n-1}^{(0)}=\operatorname{AddPosEnc}\left(\boldsymbol{w}_{t, 1: n-1}\right) .
\end{gathered}
$$

Next, the text decoder integrates $\boldsymbol{u}_{t, 1: n-1}^{(0)}$ with input speech contexts $\boldsymbol{H}_{t}^{(I)}$ and all preceding linguistic contexts $\boldsymbol{Z}_{1: t-1}^{(L)}$ using $J$ transformer decoder blocks. The $j$-th transformer decoder block creates the $j$-th hidden representation $\boldsymbol{u}_{t, n-1}^{(j)} \in \mathbb{R}^{d \times 1}$ from the lower layer inputs $\boldsymbol{u}_{1: n-1}^{(j-1)} \in \mathbb{R}^{d \times n-1}$ by

$$
\boldsymbol{u}_{t, n-1}^{(j)}=\text { TransformerDec }\left(\boldsymbol{U}_{t, 1: n-1}^{(j-1)}, \boldsymbol{H}_{t}^{(I)}, \boldsymbol{Z}_{1: t-1}^{(L)} ; \boldsymbol{\theta}_{\mathrm{dec}}\right),
$$

where TransformerDec() is a transformer decoder block that consists of a masked multi-head self-attention layer, two multi-head source-target attention layers, and a position-wise feed-forward network. In the multi-head source-target attention layers, we first use $\boldsymbol{H}_{t}^{(I)}$ then use $\boldsymbol{Z}_{1: t-1}^{(L)}$ as the source information. The predicted probabilities of the $n$-th token for the $t$-th utterance $w_{t, n}$ are calculated as

$$
P\left(w_{t, n} \mid w_{t, 1: n-1}, \boldsymbol{W}_{1: t-1}, \boldsymbol{X}_{t}, \boldsymbol{\Theta}\right)=\operatorname{Softmax}\left(\boldsymbol{u}_{t, n-1}^{(J)} ; \boldsymbol{\theta}_{\mathrm{dec}}\right)
$$

where $\operatorname{Softmax}()$ is a softmax layer with a linear transformation.

\subsection{Training}

The model parameter sets can be optimized from training datasets $\mathcal{D}=\left\{\left(\mathcal{X}^{1}, \mathcal{W}^{1}\right), \cdots,\left(\mathcal{X}^{|\mathcal{D}|}, \mathcal{W}^{|\mathcal{D}|}\right)\right\}$, where $|\mathcal{D}|$ is the number of conversation-level or discourse-level data elements in the training datasets. The $a$-th data element is represented as $\mathcal{X}^{a}=$ $\left\{\boldsymbol{X}_{1}^{a}, \cdots, \boldsymbol{X}_{T^{a}}^{a}\right\}$ and $\mathcal{W}^{a}=\left\{\boldsymbol{W}_{1}^{a}, \cdots, \boldsymbol{W}_{T^{a}}^{a}\right\}$, where $\boldsymbol{W}_{t}^{a}=$ $\left\{w_{t, 1}^{a}, \cdots, w_{t, N_{t}^{a}}^{a}\right\}$. The loss function to optimize the model parameter sets with the maximum likelihood criterion is defined as

$$
\begin{array}{r}
\mathcal{L}(\boldsymbol{\Theta})=-\sum_{a=1}^{|\mathcal{D}|} \sum_{t=1}^{T^{a}} \sum_{n=1}^{N_{t}^{a}} \sum_{w_{t, n}^{a} \in \mathcal{V}} \hat{P}\left(w_{t, n}^{a} \mid w_{t, 1: n-1}^{a}, \boldsymbol{W}_{1: t-1}^{a}, \boldsymbol{X}_{t}^{a}\right) \\
\log P\left(w_{t, n}^{a} \mid w_{t, 1: n-1}^{a}, \boldsymbol{W}_{1: t-1}^{a}, \boldsymbol{X}_{t}^{a}, \boldsymbol{\Theta}\right),
\end{array}
$$

where $\mathcal{V}$ is the vocabulary set. $\hat{P}$ represents the ground-truth probability that is 1 when $w_{t, n}^{a}=\hat{w}_{t, n}^{a}$, and 0 when $w_{t, n}^{a} \neq \hat{w}_{t, n}^{a}$. Note that $\hat{w}_{t, n}^{a}$ is the $n$-th reference token in the $t$-th utterance in the $a$-th element.

\section{LARGE-CONTEXT KNOWLEDGE DISTILLATION}

This section details our proposed large-context knowledge distillation method as an effective training method of large-context E2E-ASR models. Our key idea is to mimic the behavior of a large-context language model [9-13] pre-trained from the same training datasets. A large-context language model defines the generation probability of a sequence of utterance-level texts $\mathcal{W}=$ $\left\{\boldsymbol{W}_{1}, \cdots, \boldsymbol{W}_{T}\right\}$ as

$$
P(\mathcal{W} \mid \boldsymbol{\Lambda})=\prod_{t=1}^{T} \prod_{n=1}^{N^{t}} P\left(w_{t, n} \mid w_{t, 1: n-1}, \boldsymbol{W}_{1: t-1}, \boldsymbol{\Lambda}\right),
$$

where $\Lambda$ is the model parameter set for the model. For the network structure, we use the hierarchical text encoder and the text decoder described in Section 3.1. A loss function to optimize $\boldsymbol{\Lambda}$ is defined as

$$
\begin{array}{r}
\mathcal{L}(\boldsymbol{\Lambda})=-\sum_{a=1}^{|\mathcal{D}|} \sum_{t=1}^{T^{a}} \sum_{n=1}^{N_{t}^{a}} \sum_{w_{t, n}^{a} \in \mathcal{V}} \hat{P}\left(w_{t, n}^{a} \mid w_{t, 1: n-1}^{a}, \boldsymbol{W}_{1: t-1}^{a}\right) \\
\log P\left(w_{t, n}^{a} \mid w_{t, 1: n-1}^{a}, \boldsymbol{W}_{1: t-1}^{a}, \boldsymbol{\Lambda}\right) .
\end{array}
$$

We use the pre-trained parameter $\hat{\Lambda}$ for a target smoothing of the large-context E2E-ASR training. With our proposed large-context knowledge distillation method, a loss function to optimize $\Theta$ is defined as

$$
\begin{gathered}
\mathcal{L}_{\mathrm{kd}}(\boldsymbol{\Theta})=-\sum_{a=1}^{|\mathcal{D}|} \sum_{t=1}^{T^{a}} \sum_{n=1}^{N_{t}^{a}} \sum_{w_{t, n}^{a} \in \mathcal{V}} \tilde{P}\left(w_{t, n}^{a} \mid w_{t, 1: n-1}^{a}, \boldsymbol{W}_{1: t-1}^{a}, \boldsymbol{X}_{t}^{a}\right) \\
\log P\left(w_{t, n}^{a} \mid w_{t, 1: n-1}^{a}, \boldsymbol{W}_{1: t-1}^{a}, \boldsymbol{X}_{t}^{a}, \boldsymbol{\Theta}\right), \\
\tilde{P}\left(w_{t, n} \mid w_{t, 1: n-1}, \boldsymbol{W}_{1: t-1}, \boldsymbol{X}_{t}\right)= \\
(1-\alpha) \hat{P}\left(w_{t, n} \mid w_{t, 1: n-1}, \boldsymbol{W}_{1: t-1}, \boldsymbol{X}_{t}\right)+ \\
\alpha P\left(w_{t, n} \mid w_{t, 1: n-1}, \boldsymbol{W}_{1: t-1}, \hat{\boldsymbol{\Lambda}}\right),
\end{gathered}
$$

where $\alpha$ is a smoothing weight to adjust the smoothing term. Thus, target distributions are smoothed by distributions computed from the pre-trained large-context language model. Note that this target smoothing is regarded as an extension of label smoothing [27] to enable the use of contexts beyond utterance boundaries. 
Table 1: Experimental datasets

\begin{tabular}{|l|rrrr|}
\hline & $\begin{array}{r}\text { Data size } \\
\text { (Hours) }\end{array}$ & $\begin{array}{r}\text { Number of } \\
\text { lectures }\end{array}$ & $\begin{array}{r}\text { Number of } \\
\text { utterances }\end{array}$ & $\begin{array}{r}\text { Number of } \\
\text { characters }\end{array}$ \\
\hline \hline Train & 512.6 & 3,181 & 413,240 & $13,349,780$ \\
Valid & 4.8 & 33 & 4,166 & 122,097 \\
Test 1 & 1.8 & 10 & 1,272 & 48,064 \\
Test 2 & 1.9 & 10 & 1,292 & 47,970 \\
Test 3 & 1.3 & 10 & 1,385 & 32,089 \\
\hline
\end{tabular}

\section{EXPERIMENTS}

The effectiveness of the proposed model and method were evaluated on Japanese discourse ASR tasks using the Corpus of Spontaneous Japanese (CSJ) [28]. We divided the CSJ into a training set (Train), validation set (Valid), and three test sets (Test 1, 2, and 3). The validation set was used for optimizing several hyper parameters. The segmentation of each discourse-level speech into utterances followed a previous study [29]. We used characters as the tokens. Details of the datasets are given in Table 1.

\subsection{Setups}

We compared our proposed hierarchical transformer-based largecontext E2E-ASR model with an RNN-based utterance-level E2EASR model [3], transformer-based utterance-level E2E-ASR model [6], and hierarchical RNN-based large-context E2E-ASR model [8]. We used $40 \log$ mel-scale filterbank coefficients appended with delta and acceleration coefficients as acoustic features. The frame shift was $10 \mathrm{~ms}$. The acoustic features passed two convolution and max pooling layers with a stride of 2 , so we down-sampled them to $1 / 4$ along with the time axis. For the RNN-based models, the same setup as in previous studies were used [8]. For the hierarchical text encoder, we stacked two token-level transformer encoder blocks and two utterance-level transformer encoder blocks. For the speech encoder and text decoder, we stacked eight transformer encoder blocks and six transformer decoder blocks. The transformer blocks were created under the following conditions: the dimensions of the output continuous representations were set to 256 , dimensions of the inner outputs in the position-wise feed forward networks were set to 2,048 , and number of heads in the multi-head attentions was set to 4. In the nonlinear transformational functions, the GELU activation was used. The output unit size, which corresponds to the number of characters in the training set, was set to 3,084. We also constructed a hierarchical transformer-based large-context language model. The network structure was almost that same as our proposed hierarchical transformer-based large-context E2E-ASR model.

For the mini-batch training, we truncated each lecture to 50 utterances. The mini-batch size was set to 4 , and the dropout rate in the transformer blocks was set to 0.1. We used the Radam [30] for optimization. The training steps were stopped based on early stopping using the validation set. We also applied SpecAugment with frequency masking and time masking [31], where the number of frequency masks and time-step masks were set to 2, frequency-masking width was randomly chosen from 0 to 20 frequency bins, and timemasking width was randomly chosen from 0 to 100 frames. We also applied label smoothing [27] and knowledge distillation using a pretrained language model [24] to the utterance-level E2E-ASR models, and applied label smoothing and our proposed large-context knowledge distillation method using a pre-trained large-context language model to the large-context E2E-ASR models. Hyper-parameters were tuned using the validation set. For ASR decoding using both the utterance-level and large-context end-to-end ASR, we used a beam search algorithm in which the beam size was set to 4 .
Table 2: Comparison with conventional models

\begin{tabular}{|ll|rrr|}
\hline Model & ASR system & Test 1 & Test 2 & Test 3 \\
\hline \hline RNN [3] & Utterance-level & 8.9 & 6.7 & 7.9 \\
Transformer [6] & Utterance-level & 7.6 & 5.9 & 6.0 \\
Hierarchical RNN [8] & Large-context & 8.4 & 6.2 & 7.2 \\
Hierarchical transformer & Large-context & $\mathbf{7 . 0}$ & $\mathbf{5 . 3}$ & $\mathbf{5 . 5}$ \\
\hline
\end{tabular}

Table 3: Effect of ASR errors in preceding contexts

\begin{tabular}{|ll|rrr|}
\hline Model & Preceding contexts & Test 1 & Test 2 & Test 3 \\
\hline \hline Transformer 6] & - & 7.6 & 5.9 & 6.0 \\
Hierarchical transformer & Hypotheses & $\mathbf{7 . 0}$ & $\mathbf{5 . 3}$ & 5.5 \\
Hierarchical transformer & Oracle & $\mathbf{7 . 0}$ & $\mathbf{5 . 3}$ & $\mathbf{5 . 4}$ \\
\hline
\end{tabular}

Table 4: Effect of large-context knowledge distillation

\begin{tabular}{|c|c|c|c|c|}
\hline Model & Target smoothing & Test 1 & Test 2 & Test 3 \\
\hline Transformer 6 & - & $\overline{7.6}$ & $\overline{5.9}$ & 6.0 \\
\hline Transformer 6 & Label smoothing [27] & 7.1 & 5.1 & 5.3 \\
\hline Transformer $\overline{6}$ & KD 24 & 7.1 & 5.2 & 5.4 \\
\hline Hierarchical transformer & - & 7.0 & 5.3 & 5.5 \\
\hline Hierarchical transformer & Label smoothing [27 & 6.7 & 4.5 & 4.8 \\
\hline Hierarchical transformer & Large-context KD & 6.5 & 4.3 & 4.5 \\
\hline
\end{tabular}

\subsection{Results}

Tables 2-4 show the evaluation results in terms of character error rate (\%).Table 2 shows the results of comparing our proposed hierarchical transformer-based large-context E2E-ASR model with the above-mentioned conventional models (we did not introduce target smoothing into each model). The results indicate that the proposed model improved ASR performance compared with the transformerbased utterance-level model and RNN-based large-context model. This indicates that the large-context architecture of the proposed model can effectively capture long-range sequential contexts while retaining the strengths of the transformer. Table 3 shows the results of using oracle preceding contexts for the proposed model to reveal whether recognition errors of the preceding contexts affect ASR performance. The results indicate that using oracle contexts is comparable with using ASR hypotheses. This indicates that recognition errors in the preceding contexts rarely affect total ASR performance. Table 4 shows the results of applying label smoothing, knowledge distillation (KD in this table) and our proposed large-context knowledge distillation (large-context $\mathrm{KD}$ in this table) to the E2E-ASR models for target smoothing. The results indicate that our proposed large-context knowledge distillation effectively improved our hierarchical transformer-based large-context E2E-ASR model compared with no target smoothing and label smoothing. This confirms that our large-context knowledge distillation, which mimics the behavior of a pre-trained large-context language model, enables a largecontext E2E-ASR model to use large-contexts. These results indicate that our proposed hierarchical transformer-based large-context model with our large-context knowledge distillation method is effective in discourse-level ASR tasks.

\section{CONCLUSIONS}

We proposed a hierarchical transformer-based large-context E2EASR model and a large-context knowledge distillation method as an effective training method. The key advantage of the proposed model is that long-range sequential contexts beyond utterance boundaries can be captured while retaining the strengths of transformer-based E2E-ASR. Our large-context knowledge distillation method enables a large-context E2E-ASR model to use long-range contexts by mimicking the behavior of a large-context language model. Experimental results on discourse ASR tasks indicate that the proposed model and proposed training method effectively improves ASR performance. 


\section{REFERENCES}

[1] Geoffrey Zweig, Chengzhu Yu, Jasha Droppo, and Andreas Stolcke, "Advances in all-neural speech recognition," In Proc. International Conference on Acoustics, Speech, and Signal Processing (ICASSP), pp. 4805-4809, 2017.

[2] Kartik Audhkhasi, Bhuvana Ramabhadran, George Saon, Michael Picheny, and David Nahamoo, "Direct acoustics-to-word models for English conversational speech recognition," In Proc. Annual Conference of the International Speech Communication Association (INTERSPEECH), pp. 959-963, 2017.

[3] Dzmitry Bahdanau, Jan Chorowski, Dmitriy Serdyuk, Philemon Brakel, and Yoshua Bengio, "End-to-end attention-based large vocabulary speech recognition," In Proc. International Conference on Acoustics, Speech, and Signal Processing (ICASSP), pp. 4945-4949, 2015.

[4] Liang Lu, Xingxing Zhang, Kyunghyun Cho, and Steve Renals, "A study of the recurrent neural network encoder-decoder for large vocabulary speech recognition," In Proc. Annual Conference of the International Speech Communication Association (INTERSPEECH), pp. 3249-3253, 2015.

[5] Ashish Vaswani, Noam Shazeer, Niki Parmar, Jakob Uszkoreit, Llion Jones, Aidan N. Gomez, Lukasz Kaiser, and Illia Polosukhin, "Attention is all you need," In Proc. Advances in Neural Information Processing Systems (NIPS), pp. 5998-6008, 2017.

[6] Linhao Dong, Shuang Xu, and Bo Xu, "Speech-Transformer: A norecurrence sequence-to-sequence model for speech recognition," In Proc. International Conference on Acoustics, Speech, and Signal Processing (ICASSP), pp. 5884-5888, 2018.

[7] Suyoun Kim and Florian Metze, "Dialog-context aware end-to-end speech recognition," In Proc. Spoken Language Technology Workshop (SLT), pp. 434-440, 2018.

[8] Ryo Masumura, Tomohiro Tanaka, Takafumi Moriya, Yusuke Shinohara, Takanobu Oba, and Yushi Aono, "Large context end-to-end automatic speech recognition via extension of hierarchical recurrent encoder-decoder models," In Proc. International Conference on Acoustics, Speech, and Signal Processing (ICASSP), pp. 5661-5665, 2019.

[9] Rui Lin, Shujie Liu, Muyun Yang, Mu Li, Ming Zhou, and Sheng Li, "Hierarchical recurrent neural network for document modeling," In Proc. Conference on Empirical Methods in Natural Language Processing (EMNLP), pp. 899-907, 2015.

[10] Tian Wang and Kyunghyun Cho, "Larger-context language modelling with recurrent neural network," In Proc. Annual Meeting of the Association for Computational Linguistics (ACL), pp. 1319-1329, 2016.

[11] Ryo Masumura, Tomohiro Tanaka, Atsushi Ando, Hirokazu Masataki, and Yushi Aono, "Role play dialogue aware language models based on conditional hierarchical recurrent encoder-decoder," In Proc. Annual Conference of the International Speech Communication Association (INTERSPEECH), pp. 1259-1263, 2018.

[12] Ryo Masumura, Tomohiro Tanaka, Atsushi Ando, Hosana Kamiyama, Takanobu Oba, Satoshi Kobashikawa, and Yushi Aono, "Improving conversation-context language models with multiple spoken language understanding models," In Proc. Annual Conference of the International Speech Communication Association (INTERSPEECH), pp. 834838, 2019.

[13] Ryo Masumura, Mana Ihori, Tomohiro Tanaka, Itsumi Saito, Kyosuke Nishida, and Takanobu Oba, "Generalized large-context language models based on forward-backward hierarchical recurrent encoder-decoder models," In Proc. Automatic Speech Recognition and Understanding Workshop (ASRU), pp. 554-561, 2019.

[14] Longyue Wang, Zhaopeng Tu, Andy Way, and Qun Liu, "Exploiting cross-sentence context for neural machine translation," In Proc. Conference on Empirical Methods in Natural Language Processing (EMNLP), pp. 2826-2831, 2017.

[15] Sameen Maruf and Gholamreza Haffari, "Document context neural machine translation with memory networks," In Proc. Annual Meeting of the Association for Computational Linguistics (ACL), pp. 1275-1284, 2018.
[16] Iulian V. Serban, Alessandro Sordoni, Yoshua Bengio, Aaron Courville, and Joelle Pineau, "Building end-to-end dialogue systems using generative hierarchical neural network models," In Proc. AAAI Conference on Artificial Intelligence (AAAI), pp. 3776-3783, 2016.

[17] Mana Ihori, Akihiro Takashima, and Ryo Masumura, "Large-context pointer-generator networks for spoken-to-written style conversion," In Proc. International Conference on Acoustics, Speech, and Signal Processing (ICASSP), pp. 8184-8188, 2020.

[18] Jiacheng Zhang, Huanbo Luan, Maosong Sun, Feifei Zhai, Jingfang $\mathrm{Xu}$, Min Zhang, and Yang Liu, "Improving the transformer translation model with document-level context," In Proc. Conference on Empirical Methods in Natural Language Processing (EMNLP), pp. 533-542, 2018.

[19] Xin Tan, Longyin Zhang, Deyi Xiong, and Guodong Zhou, "Hierarchical modeling of global context for document-level neural machine translation," In Proc. Conference on Empirical Methods in Natural Language Processing (EMNLP), pp. 1576-1585, 2019.

[20] Yang Liu and Mirella Lapata, "Hierarchical transformers for multidocument summarization," In Proc. Annual Meeting of the Association for Computational Linguistics (ACL), pp. 5070-5081, 2019.

[21] Mingkun Huang, Yongbin You, Zhehuai Chen, Yanmin Qian, and Kai Yu, "Knowledge distillation for sequence model," In Proc. Annual Conference of the International Speech Communication Association (INTERSPEECH), pp. 3703-3707, 2018.

[22] Ho-Gyeong Kim, Hwidong Na, Hoshik Lee, Jihyun Lee, Tae Gyoon Kang, Min-Joong Lee, and Young Sang Choi, "Knowledge distillation using output errors for self-attention end-to-end models," In Proc. International Conference on Acoustics, Speech, and Signal Processing (ICASSP), pp. pp.6181-6185, 2019.

[23] Raden Mu'az Mun'im, Nakamasa Inoue, and Koichi Shinoda, "Sequence-level knowledge distillation for model compression of attention-based sequence-to-sequence speech recognition," In Proc. International Conference on Acoustics, Speech, and Signal Processing (ICASSP), 2019.

[24] Ye Bai, Jiangyan Yi, Jianhua Tao, Zhengkun Tian, and Zhengqi Wen, "Learn spelling from teachers: Transferring knowledge from language models to sequence-to-sequence speech recognition," In Proc. Annual Conference of the International Speech Communication Association (INTERSPEECH), pp. 3795-3799, 2019.

[25] Hayato Futami, Hirofumi Inaguma, Sei Ueno, Masato Mimura, Shinsuke Sakai, and Tatsuya Kawahara, "Distilling the knowledge of BERT for sequence-to-sequence ASR," arXiv:2008.03822 2020.

[26] Zhouhan Lin, Minwei Feng, Cicero Nogueira dos Santos, Mo Yu, Bing Xiang, Bowen Zhou, and Yoshua Bengio, "A structured self-attentive sentence embedding," In Proc. ICLR, 2017.

[27] Christian Szegedy, Vincent Vanhoucke, Sergey Ioffe, Jon Shlens, and Zbigniew Wojna, "Rethinking the inception architecture for computer vision," In Proc. IEEE conference on Computer Vision and Pattern Recognition (CVPR), pp. 2818-2826, 2016.

[28] Kikuo Maekawa, Hanae Koiso, Sadaoki Furui, and Hitoshi Isahara, "Spontaneous speech corpus of Japanese," In proc. International Conference on Language Resources and Evaluation (LREC), pp. 947-952, 2000.

[29] Takaaki Hori, Shinji Watanabe, Yu Zhang, and William Chan, "Advances in joint CTC-attention based end-to-end speech recognition with a deep CNN encoder and RNN-LM," In Proc. Annual Conference of the International Speech Communication Association (INTERSPEECH), pp. 949-953, 2017.

[30] Liyuan Liu, Haoming Jiang, Pengcheng He, Weizhu Chen, Xiaodong Liu, Jianfeng Gao, and Jiawei Han, "On the variance of the adaptive learning rate and beyond," In Proc. International Conference on Learning Representations (ICLR), 2020.

[31] Daniel S. Park, William Chan, Yu Zhang, Chung-Cheng Chiu, Barret Zoph, Ekin D. Cubuk, and Quoc V. Le, "SpecAugment: A simple data augmentation method for automatic speech recognition," In Proc. Annual Conference of the International Speech Communication Association (INTERSPEECH), pp. 2613-2617, 2019. 